\title{
Using PCR to Compare the Expression of CDR1, CDR2, and MDR1 in Candida Albicans Isolates Resistant and Susceptible to Fluconazole
}

Nahid Ariana (MSc)

Department of Microbiology, Faculty of Biological Sciences, Islamic Azad University, Tonekabon Branch, Iran

Ali Nazemi (PhD)

Department of Genetics, Faculty of Biological Sciences, Islamic Azad University, Tonekabon Branch, Iran

Ayatollah Nasrollahi Omran (PhD)

Department of Microbiology, Faculty of Biological Sciences, Islamic Azad University, Tonekabon Branch, Iran

Corresponding Author: Nahid Ariana

Email: Ariana-nahid@yahoo.com

Tel: +989188396936

Address: Faculty of Biological Sciences, Islamic Azad University, Tonekabon Branch, Iran

Received: Jan 112014

Revised: Sep 62014

Accepted: Sep 82014

\begin{abstract}
Background and Objectives: More Candida albicans strains are reported resistant to fluconazole in patients with AIISS, cancer and organ recipients. Fluconazole resistance can be attributed to changes in pathways of sterol biosynthesis, mutation in or overexpression of ERG1l and the expression of CDR1, CDR2, and MDRL. This study aimed to compare the expression of CDR1, CDR2, and MDR1 in C. albicans resistant and susceptible to fluconazole.

Methods: MIC testing for fluconazole was performed on C. albicans isolates isolated from patients with oral and vaginal candidiasis to determine resistant and susceptible strains. Then real time PCR was performed on the resistant and susceptible isolates and the expression of CDR1, CDR2, and MDR1 was compared in C. albicans.

Results: 0f 46 Candida albicans isolates, 20 susceptible isolates, 12 semi-susceptible isolates and 14 resistant isolates were identified by MIC. After real time PCR was performed, Candida albicans isolates susceptible to fluconazole showed moderate expression of CDR1, CDR2, and MIDRl genes, while resistant isolates showed slight or no expression.

Conclusion: Increased expression of CDR1, CDR2, and MIDR had less and insignificant role in resistance to fluconazole.

Keywords: Candida Albicans, Gene Expression, Real time PCR method.
\end{abstract}




\section{INTRODUCTION}

Candidiasis is one of the most important and common opportunistic fungal diseases in humans caused by the fungus Candida albicans (1). Candidemia can be observed in patients with immune deficiency and newborns etc. $(2,3)$. Increased fungal infections, patients infected with HIV, cancer patients undergoing chemotherapy and suffering neutropenia, patients with immune deficiency or under treatment with broad-spectrum antibiotics and glucocorticoids and dialysis patients are more susceptible for infection. C. albicans is the fourth cause of sepsis in the United States $(4,5)$. The most common antifungal medications are classified as: ergosterol synthesis inhibitor, polyenes and 5-FC. Allylamines (thiocarbamates) and azoles fall in the first class $(6,7)$. Performing tests to determine the susceptibility of the disease agent to antifungal medications is necessary for successful treatment of patients with dangerous fungal infections. Triazole antifungal agents are usually used for candidiasis because of their high therapeutic index (8). Medication resistance to azoles is a major concern. Resistant strains showed changes in the quantity and quality of target enzymes, reduced information about the target, or a combination of the two (9). In AIDS patients who were under treatment with fluconazole for a long time, clinical resistance showed isolated C. albicans strains and reduced susceptibility to fluconazole in vitro. There are different molecular mechanisms for fluconazole resistance in $C$. albicans including changes in sterol biosynthesis pathways, mutation in or overexpression of ERG11 gene, increased expression of genes encoding efflux pumps: highly homologous CDR1 and CDR2 genes (drug resistance of Candida) and MDR1 (multidrug resistance) and FLU1 (fluconazole resistance) and multiple mechanisms due to interaction between different mechanisms ( 8 , 10-14). This study aimed to investigate the expression of MDR1, CDR2, and CDR1 in fluconazole-resistant and -susceptible strains of C. albicans using RT-PCR and compare the expression of these genes in susceptible and resistant isolates.

\section{MATERIAL AND METHODS}

A total of 72 clinical isolates were collected and analyzed from patients suspected of having oral and vaginal candidiasis with symptoms such as itching and burning in the vaginal area or recurrent oral thrush. The samples were obtained from people who attended health clinics in Tonekabon, Sari, and Rasht from December 2012/January 2013 to May/June 2013.

After samples were cultured by sterile swab on Sabouraud dextrose agar medium (MERCK Company, Germany) containing chloramphenicol (antibacterial antibiotic) (Daru Sina), the growth of yeast colonies was observed and microscopic examination was conducted to identify strains by germ tube test, chlamydospore formation in corn meal agar medium containing $0.5 \%$ Tween 80 and API sugar absorption test $(14,15)$.

Drug susceptibility testing (MIC)

The following steps were carefully conducted to perform the test $(11,16)$ :

A) Preparation of the fungal suspension: a suspension of each new culture (48 hours) of $C$. albicans was prepared by sterile physiological saline and a concentration of $1.5 \times 10^{8}$ cells was prepared from yeasts with half McFarland. Ten dilution series from 1.128 to $1.025 \mu \mathrm{g} / \mathrm{ml}$ of fluconazole were prepared. In this study, broth microdilution method was used according to NCCLS M27A. For fluconazole, sequential dilutions twice as much as medication stock were prepared with sterile physiological saline in ten tubes. The highest and lowest dilutions of the medication were prepared, 1.128 and 1.025 $\mu \mathrm{g} / \mathrm{ml}$, respectively, and $100 \mu \mathrm{l}$ was poured into the wells of 24-well microplates. Then $100 \mu \mathrm{l}$ of RPMI1640 medium was added to each well. The yeast suspension of $1.5 \times 10^{8} \mathrm{cfu} / \mathrm{m}$ was prepared for each well in a tube and was added to microplates by calculating the amount needed for each well. The well containing growth medium without drug was also used as control $(9,11,16)$. Microplates were incubated separately in the shaker incubator at $35^{\circ} \mathrm{C}$ for 48 hours. Then $10 \mu \mathrm{l}$ of contents of each well was inoculated to plates containing Sabouraud dextrose agar with chloramphenicol $(\mathrm{Sc})$ and was spread by a loop and the plates were incubated for 48 hours at $35^{\circ} \mathrm{C}$. The values of minimum inhibitory concentration (MIC) of fluconazole were calculated by counting the number of colonies grown on each dilution compared to the control group. For fluconazole, C. albicans grown in the concentration $\geq 64$ $\mu \mathrm{g} / \mathrm{ml}$ was resistant to fluconazole, colonies grown in the concentration $\leq 8 \mu \mathrm{g} / \mathrm{ml}$ were susceptible to fluconazole, and colonies at concentrations of $16-32 \mu \mathrm{g} / \mathrm{ml}$ were dosedependent susceptible $(9,11,16)$. 
After separation of susceptible and resistant isolates of C. albicans, each of the isolates was cultured in Sabouraud dextrose broth medium and was incubated in the incubator shaker at $37{ }^{\circ} \mathrm{C}$ for 24 hours. Then fresh medium was added at a ratio of 1:1 to the sample grown in the liquid medium and incubated again in the incubator shaker for 18 hours. Susceptible and resistant samples were examined in the spectrophotometer and their OD was read at wavelength $600 \mathrm{~nm}$. When both samples showed $\mathrm{OD}=1.2$ at wavelength $600 \mathrm{~nm}, 1.5 \mathrm{ml}$ of samples were poured into a sterile microtube and RNA was extracted by two methods including RNA extraction kit (INTRON Company) and phenol-chloroform extraction. To ensure the accuracy of RNA extraction, a little amount of the extracted RNA was loaded on agarose gel after extraction and its band was observed. To perform two-step RT REAL-TIME PCR, a primer of (20 pmol (METABION GERMANY)) $1 \mu \mathrm{l}$, dNTPmix $2 \mu \mathrm{l}$, RT BUFFER (5X) $5 \mu \mathrm{l}$, RT ENZYME (THERMO SCIENTIFIC EP0441USA) $2 \mu$, RNA Templete $15 \mu \mathrm{l}$ was mixed and the volume was reached to $25 \mu \mathrm{l}$. After the target RT MIX was prepared, it was placed in a thermocycler for one hour at $42^{\circ} \mathrm{C}$. Then the mixture were added to the PCR MIX and placed in the RT PCR machine. To prepare PCR MIX, the volume of Taq polymerase enzyme $0.4 \mu \mathrm{l}$, Taq buffer (10X) $2.5 \mu$ l, Primer $\operatorname{mix}(20$ pmol $) 1 \mu \mathrm{l}$, dNTP mix $(10 \mathrm{~m} \mathrm{M}) 0.5 \mu \mathrm{l}$, $\mathrm{MgCl} 2(50 \mathrm{~m} \mathrm{M}) 1 \mu \mathrm{l}$, Cyto9 $1 \mu \mathrm{l}, \mathrm{H} 2 \mathrm{O} 17 \mu \mathrm{l}$, RNA Templete $2 \mu \mathrm{l}$ was reached to volume of $25 \mu 1$. The values are used for positive and negative control; the only difference is that $2 \mu \mathrm{l}$ water was added in the negative control instead of $2 \mu \mathrm{l}$ sample. Then positive and negative control microtubes were placed in a thermocycler and after a phase of denaturation at $95^{\circ} \mathrm{C}$ for $5 \mathrm{~min}$, three-phase temperature cycles $95^{\circ} \mathrm{C}$ and $60{ }^{\circ} \mathrm{C}$ and $72{ }^{\circ} \mathrm{C}$ were repeated for 30 seconds for 35 cycles and the final cycle was performed at $72{ }^{\circ} \mathrm{C}$ for five minutes. These temperature cycles were used for all CDR1, CDR2, and MDR1 genes, and the annealing temperature was reduced to $52{ }^{\circ} \mathrm{C}$ only for CDR2 due to low melting temperature of the primer. After these steps were completed, RT PCR machine showed the curves of gene expression in the positive and negative samples. Post PCR was performed to determine the accuracy of the reaction. PCR products of genes and negative controls on agarose gel were $1.5 \%$, and the marker was loaded to show the size of gene segments.

\section{RESULTS}

From a total of 72 samples collected from patients suspected of having oral and vaginal candidiasis, 51 samples were identified as candidiasis samples. After culturing, examining, performing germ tube test, and chlamydospore formation on corn meal agar containing $0.5 \%$ Tween 80, only 46 samples were found to be $C$. albicans. MIC testing was performed by broth microdilution according to the standard method of NCCLS M27A on 46 Candida albicans isolates detected by antifungal agents susceptibility testing and 20 samples were found susceptible, 12 were semi-susceptible and 14 were resistant. A standard sample of $C$. albicans prepared from Sari Medical Faculty was also used as control to confirm the PCR of resistant samples. After RT PCR was performed, $C$. albicans isolates susceptible to fluconazole showed moderate expression of CDR1, CDR2, and MDR1 genes, while C. albicans isolates resistant to fluconazole showed little or no expression of these genes. According to figures obtained from RT PCR performed for CDR1, CDR2, and MDR1, the expression of CDR1, MDR1 in susceptible $C$. albicans isolates was more than their expression in resistant isolates, and CDR2 had a very low or zero expression in C. albicans isolates susceptible to fluconazole and in isolates resistant to fluconazole. In these tables, resistant sample was considered the standard sample (Table1).

Table1- Primers used in the reaction $R T R E A L-T I M E P C R(11)$

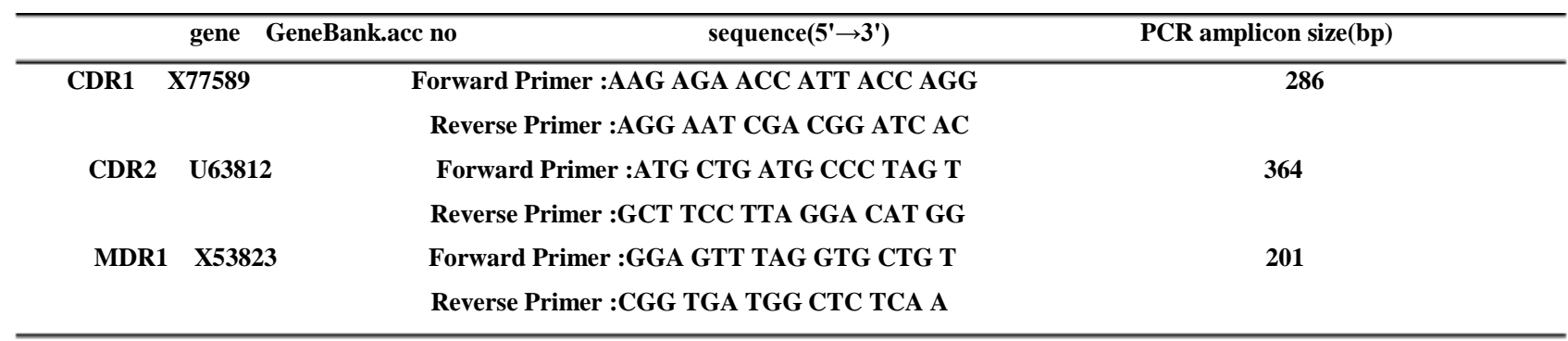




\section{DISCUSSION}

The difference in the expression of CDR1, CDR2, and MDR1 in susceptible and resistant strains of $C$. albicans shows the role of these genes in the development of resistance to fluconazole. The resistance of $C$. albicans to fluconazole may be attributed to increased expression of or mutation in ERG11 and changes in the ergosterol biosynthesis or interactive mechanisms than to the increased expression of CDR1, CDR2, and MDR1. The lack of expression of genes appears to cause resistance, and their expression appears to cause susceptibility. Given the products of the above genes, the plasma membrane efflux pumps, it appears that fluconazole disrupts the function of these pumps and inhibiting their function leads to susceptibility to antibiotics without lethal effect. No clear evidence has been provided yet that efflux pumps of $C$. albicans bind to antifungal drugs and transporting them. Transporting fluconazole in $C$. albicans secretory vesicles by membrane proteins of MDR1P, CDR2P, and CDR1P is a main reason for resistance to azoles, especially fluconazole in C. albicans (17). Similar findings have not been reported for increased expression of $C$. albicans CDR1; these observations suggest that the development of drug resistance may create new vulnerable sites which might cause preferential attacks to strains resistant to fluconazole (9). Some isolates resistant to fluconazole show reduced pathogenicity, but pathogenicity may remain unchanged or increase. Recently, $C$. albicans with resistant mutations has been reported to have reduced adaptation compared to susceptible isolates in hosts. An important study about the intrinsic resistance of $C$. albicans to fluconazole also shows that biofilm growth of an organism is independent from the expression of efflux pumps (18). MIC revealed 20 C. albicans isolates susceptible to fluconazole, 12 dosedependent susceptible and 14 resistant isolates. Lee reported seven samples as susceptible, three samples as dose-dependent susceptible and two samples as resistant among the 12 isolates of $C$. albicans in his study, which is consistent with our study (16). In this study, RT PCR based probe-free with SYTO-9 fluorescence dye was used. This method was used because of its excellent receivers, high throughput in sequencing and making hybrid of melted information, high specificity and close-TIOB reaction that does not need Post PCR. Lee stated that CDR1 and MDR1 show high expression in all C. albicans isolates despite fluconazole response in vitro in the presence of fluconazole, but blood samples and mucus showed reduced susceptibility to fluconazole in vivo. The results of the abovementioned study about the expression of CDR1 and MDR1 were similar to our findings (16). Sanglard showed that some resistant $C$. albicans isolates had 10-fold increase in CDR1, but had no increased expression in other isolates. This study differs from our study in that all samples in our study show decreased expression in resistant isolates. Sanglard attributed resistance to fluconazole to energy-dependent pumps of BEN (12). Silva used AP-PCR method to evaluate resistant and susceptible strains of $C$. albicans that showed no specific results, while we used RT PCR method with high specificity and close-TIOB reaction in our experiments (15). Bhandri stated that PLAT FORM methods including RT PCR and NASBA are appropriate for molecular diagnosis of resistance to antifungal agents. This is very similar to our study and RT PCR technique was also used in our experiments (6). Ghannum attributed $C$. albicans resistance to fluconazole to the increased expression of CDR1, CDR2, and MDR1 that changes the ergosterol biosynthesis through a mutation in the target enzyme, sterol 14-demethylase. Some resistant strains, MDR1 is not transcribed in vitro or is transcribed with defects. Our results are not consistent with this study (9). Frade compared the expression of CDR1, CDR2, and MDR1 with the expression of housekeeping gene (ACT1), while we compared the expression of these genes in susceptible and resistant samples. Furthermore, this paper preferred RTLIGHTCYCLER PCR to determine $C$. albicans mRNA to other traditional methods such as northern hybridization method, and reported its advantages, which concurs with our study (11). Song stated that mutations of T916C and G487T in the genome of $C$. albicans led to increased expression of CDR1 and CDR2, but mutations increased the expression of MDR1 only in some isolates. The mentioned study is fairly consistent with our study as our isolates lacked mutation and consequently these genes had lower expression (13).

\section{CONCLUSION}

Given that resistance to fluconazole in $C$. albicans is attributed to several mechanisms, including increased expression of ERG11, mutation in this gene, changes in ergosterol biosynthesis and increased expression of CDR1, 
CDR2, and MDR1, other reasons for resistance mentioned above played a greater role in the resistance of $C$. albicans isolates collected in this study, and increased expression of CDR1, CDR2, and MDR1 had a lower and insignificant role in resistance to fluconazole. Increased expression of or mutation in ERG11 and changes in the ergosterol biosynthesis or interactive mechanisms may have higher priority than increased expression of CDR1, CDR2, and MDR1 in the resistance of $C$. albicans to fluconazole. Explanation of regulating pathways

\section{REFERENCES}

1. Guarro J, Gefne J, Stchingel AM. Development in fungal taxonomy.Clin Microbiol Rev. 1999; 12(3): 454-500.

2. Wisplinghof $\mathrm{H}$, Bischoff $\mathrm{T}$, Tallent SM, Seifert $\mathrm{H}$, Wenzel RP. Nosocomial bloodstream infections in US hospitals: analysis of 24,179 cases from a prospective nationwide surveillance study. Clin Infect Dis. 2004; 39(3): 309-317.

3. Beck-Sague CM, Jarvis WR. Secular trends in the epidemiology of nosocomial fungal infections in the United States 1980-1990. National Nosocomial Infections Surveillance System. J Infect Dis. 1993; 167: 1247-1251.

4. Hajjeh AR, Sofair AN, Harrison LH, Lyon GM, Arthington-Skaggs BA, Mirza SA, et al. Incidence of Bloodstream Infections Due to Candida apecies and In Vitro Susceptibilities of Isolated from 1998 to2000 in a Population_based active surveillance program. J Clin Microbiol. 2004; 42(4): 1519-1527.

5. Perea S, Patterson FT. Anti fungal Resistance in Pathogenic Fungi. Clin Infect Dis. 2002; 35(9): 1073-80.

6. Bhanderi BB, Yadav MM, Roy A. Antifungal Drug Resistance Concerns for Veterinarians. Veterinary World. 2009; 2(5): 204-207.

7. Ruhnke M, Hartwig K, Kofla G. New option for treatment of Candidemia in critically ill patient. Clin microbial infect. 2008; 14(Suppl.4): 46-54.

8. Sanglard D. Resistance and tolerance mechanisms to antifungal drug in fungal pathogens. Mycologist. 2003; 17(2): 74-78.

9.Ghannoum AM, Rice BL. Antifungal Agents:Mode of Action,Mechanisms of Resistance and Correlation of These Mecanisms With Bacterial Resistance. Clinical Microbiology Review.1998; 12(4): 501-517.

10. Metwally L, Hogg GT, Coyle VP, Hay JR, Hedderwick $\mathrm{S}$, McCloskey $\mathrm{B}$, et al. Rapid differentation between fluconazole_sensitive and resistant species of Candida directly from positive blood-culture bottles by real-time PCR. Journal of Medical Microbiology. 2007; 56(Pt 7): 964-970. which control the expression of these genes may be a fundamental relationship for having a close partnership with this activity and overcoming resistance.

\section{ACKNOWLEDGEMENT}

Hereby, I greatly appreciate the cooperation and assistance provided by Dr. Ali Nazemi who helped me in all stages of this research.

\section{CONFLICT OF INTEREST}

The authors declare no conflict of interest between them.

11. Frade JP, David W, Warnock, Beth A. ArthingtonSkaggs. Rapid quantification of drug resistance gene expression in Candida albicans by reverse transcriptase light cycler PCR and fluerscent probe hybridization. journal of clinical microbiology. 2004; 42(5): 2058-209.

12. Sanglard D, Kuchler K, Ischer F, Pagani jL, Monod M, Bille J. Mechanisms of Resistance to Azole Antifungal Agents in Candida albicans Isolates from AIDS Patients Involve Specific Multidrug Transporters. Antimicrobial agent and chemotherapy.1995; 39(11): 2378-2386.

13. Song wj, Shin HJ, Kee JS, Kim HS, Shin GM, Suh PS, Ryang WD. Expression of CgCDR1,Cg CDR2, and CgERG11 in Candida glabrata biofilms formed by bloodstream isolates. Medical Mycology. 2009; 47(5): 545548 .

14. Morschhauser J. The genetic basis of fluconazole resistance development in Candida albicans. Biochimica et Biophysica Acta. 2002; 1587(2-3): 240-248.

15. Silva M, Costa RM, Miranda TBA, Fernandes FLO, Costa RC, Paula RC. Evaluation of etest and macrodillution broth method for antifungal susceptibility testing of Candida sp strains isolated from oral cavities of aids patients. Rev Inst Med trop S Paulo. 2002; 44(3): 121125.

16. Lee MK, Williams EL, Warnock WD, ArthingtonSkaggs AB. Drug Resistance genes and trailing growth in Candida albicans isolates. Journal of antimicrobial chemotherapy. 2004; 53(2): 217-224

17. Basso RL, Gast EC, Mao Y, Wong B. Fluconazole Transport into Cndida albicans Secretory Vesicle by the Membrane Proteins Cdrlp,Cdr2p,and Mdrlp. Eukaryotic cell. 2010; 9(6): 960-970.

18. Watamoto T, Samaranayake LP, Egusa, Yatani H, Seneviratne CJ. Transcriptional regulation of drugresistance genes in Candida albicans biofilms in response to antifungals. journal of medical microbiology. 2011; 60: 1241-1247. 